\title{
MAPEAMENTO DE PROCESSOS: FUNDAMENTOS, FERRAMENTAS E CASO EM UMA OPERAÇÃO LOGÍSTICA
}

\author{
Thaís Cristina C.S. Azevedo \\ Departamento Engenharia Industrial, PUC-Rio \\ thais.tccsa@gmail.com \\ Ana Luisa Alves Teixeira \\ Departamento Engenharia Industrial, PUC-Rio \\ analuisa.teixeira@hotmail.com \\ Luiz Felipe Roris Rodriguez Scavarda do Carmo \\ Departamento Engenharia Industrial, PUC-Rio \\ lf.scavarda@puc-rio.br \\ Luiza Fiorencio \\ Instituto Tecgraf, PUC-Rio \\ luiza.fiorencio@gmail.com
}

\begin{abstract}
Resumo
No atual ambiente complexo em que as organizações estão inseridas, a gestão dos processos tem sido importante para a manutenção da competitividade das empresas. O mapeamento de processos é uma ferramenta aliada das organizações na gestão de seus processos e os benefícios originados de sua utilização são diversos. O objetivo deste estudo foi o de apresentar esta importante ferramenta de gestão de processos, alguns mapas que auxiliam o trabalho de mapear processos e, a utilização dos mapas sugeridos em um caso real na área de logística de uma empresa. A utilização desta ferramenta ajuda as organizações a melhorar continuamente seus processos e, deve ser realizada pelas empresas sempre que necessário.

Palavras-Chaves: Processos, SIPOC, Mapa de relacionamento, Mapa funcional de processos, Fluxograma, Logística.
\end{abstract}

\begin{abstract}
Nowadays, the organizations are inserted in a complex environment and the processes management has been important to maintain the competitiveness of the enterprises. Mapping process are an ally tool of organizations in the management of its processes and the benefits arising from their use are many. The aim of this study was to present this important process management tool, some maps that help the work of mapping processes and the use of these maps suggested in a real case in the logistics department of a company. The use of this tool helps organizations to continuously improve their processes and should be performed by companies whenever it is necessary.
\end{abstract}

Keywords:

Processes, SIPOC, Relationship map, Functional map processes, Flow Chart, Logistics. 


\section{INTRODUÇÃO}

Atualmente as empresas vêm enfrentando mudanças constantes impulsionadas por questões políticas, econômicas e ambientais, pelo rápido avanço da tecnologia da informação e pelos fatores de competitividade determinados pelo consumidor (Zeynep et. al., 2014). Porém esse ambiente no qual as organizações estão inseridas pode apresentar grandes oportunidades de crescimento para as empresas existentes e para novos mercados. Buscando atender as pressões competitivas geradas por esse ambiente, as organizações precisam reajustar suas estratégias de negócios, cadeias de suprimento, ferramentas tecnológicas e operações (Alomar e Pasek, 2014). As organizações são majoritariamente compostas por processos e conseguem gerir com maior eficácia e eficiência seus negócios quando orientadas e focadas nos seus processos (Madison, 2005). O processo de negócio é o conjunto de atividades estruturadas e relacionadas, projetadas para produzir um output (produto ou serviço) específico para um cliente ou mercado (Davenport, 1990). Nesse contexto o conceito de gestão de processos tem sido importante para a manutenção da competitividade das organizações. A gestão de processos é uma abordagem estruturada que visa a melhoria do desempenho dos processos de negócio de uma organização, ao centrar esforços disciplinados no desenho e na execução de seus processos (Hammer, 2002). Esta filosofia de gestão voltada aos processos de uma empresa e o conjunto de ferramentas desenvolvidas a partir desta filosofia foram introduzidas principalmente por dois americanos, W. Edwards Deming e Joseph M. Juran, por meio do conceito de Total Quality Management após a Segunda Guerra Mundial (Madison, 2005). A gestão de processos é uma parte importante também em projetos de implantação de mudanças ou de reengenharia dos negócios de uma empresa, e exerce influência no sucesso desses projetos ao garantir a aderência dos processos de negócio da organização ao ambiente em que ela está inserida (Trkman, 2010).

O mapeamento de processos é uma ferramenta gerencial e de comunicação essencial para líderes e organizações na promoção de melhorias e na implantação de novos processos, ou seja, para a gestão de processos das organizações. Existem diversos tipos de mapas com diferentes características e finalidades específicas. O que é capturado pelo mapeamento dependerá diretamente da técnica adotada (Biazzo, 2002) e, o uso de diferentes ferramentas de mapeamento auxilia os envolvidos no processo a atingir uma melhor performance (Greasley, 2006). Uma importante ferramenta é o SIPOC (Supplier, Input, Process, Output e Customer, em português: fornecedor, entrada, processo, saída e cliente). O SIPOC auxilia a construção dos mapas de processos. Este artigo irá contemplar três deles: o relationship map, o cross-functional process map e o flowchart.

Neste contexto, o objetivo deste artigo é o de apresentar o SIPOC e estes três tipos de mapa, para, em seguida, conduzir um caso real em uma empresa de telecomunicações, ilustrando o uso combinado da metodologia do flowchart com a do cross-functional map.

Este artigo está dividido em quatro seções, sendo esta primeira introdutória. A seção dois apresenta as razões para mapear processos e quatro importantes ferramentas que podem auxiliar nesta tarefa. A seção três apresenta o uso de uma combinação de mapas em um caso real. A quarta e última seção apresenta as conclusões do estudo. 


\section{MAPEAMENTO DE PROCESSOS: MOTIVAÇÃO E FERRAMENTAS PRÁTICAS}

Mapear um processo envolve a identificação, documentação, análise e desenvolvimento do mesmo (Anjard, 1998) formalizando em uma linguagem comum a todos da organização, o entendimento das etapas que o compõem (Damelio, 2011). Essa ferramenta desempenha o papel essencial de estimular os processos existentes, criando oportunidades de melhoria de desempenho organizacional ao identificar interfaces críticas e, sobretudo, criando bases para implantação de novas tecnologias de informação e de integração empresarial como por exemplo os ERP's (Enterprise resource planning). Neste tipo de projeto, o mapeamento de processos auxilia no desenho dos atuais processos de negócio das organizações e de como estes deveriam ser com a implantação do software, apoiando assim a transição das organizações durante o projeto (Okrent e Vokurka, 2004).

Esta análise estruturada dos processos permite ainda, reduzir custos no desenvolvimento de produtos e serviços e, de falhas de integração entre sistemas, além de possibilitar um melhor entendimento dos processos atuais e a eliminação ou simplificação dos processos que necessitam de mudanças (Damelio, 2011).

$\mathrm{O}$ mapeamento de processos também ganha importância por sua função de registro e documentação histórica dos procedimentos realizados na organização. Muitas organizações documentam o fluxo de trabalho dos seus processos por ações ou decisões que são faladas, outras documentam atividades coordenadas e sequenciadas (Kettinger et. al., 1998).

Mapear processos é fundamental na verificação de como funcionam os componentes de um sistema, na análise de sua eficácia e na identificação de suas deficiências. Segundo Kalman (2002) o mapeamento de processos é simultaneamente uma ferramenta analítica e uma metodologia de intervenção processual que visa a melhoria de performance e a redução dos erros de um processo. $\mathrm{O}$ autor define esta metodologia em sete etapas. Antes de iniciar o projeto, a seleção do processo crítico a ser mapeado, a definição de um objetivo específico e mensurável a ser alcançado e, o estabelecimento do comprometimento necessário pela gerência com o projeto são questões a serem abordadas. A primeira etapa do mapeamento é a construção do mapa do processo. Ou seja, as principais atividades que o compõe são identificadas e organizadas pela sua ordem de ocorrência. A segunda etapa consiste na identificação dos pontos de atenção no processo como gargalos e atividades com alta probabilidade de erros. A terceira etapa ocorre com a categorização e priorização dos pontos de atenção identificados. Na quarta etapa, os pontos de atenção selecionados como prioridades são analisados a fim de identificar as causas-raiz dos problemas que podem ser gerados. Na quinta etapa, o autor aborda a atividade de redesenho do processo, enfatizando a eliminação das atividades ineficientes e redundantes e indica a necessidade de redesenho do processo por mais de uma vez, assim o mesmo tende a ser bastante simplificado. A sexta etapa consiste na apresentação do novo processo à gerência e na determinação de métricas que serão utilizadas para avaliar a melhoria de performance conseguida com novo processo. A sétima e última etapa ocorre com a implantação e medição de desempenho do processo redesenhado.

A linguagem gráfica com a qual os mapas de processos são apresentados permite expor os detalhes dos processos de modo gradual e controlado, encoraja a concisão e a precisão na descrição do processo, foca a atenção nas interfaces do processo e, fornece uma análise dos processos consistente com a linguagem do projeto (Damelio, 2011).

A seguir são apresentados a ferramenta SIPOC e três importantes tipos de 
mapas de processos, tendo como base o trabalho de Hunt (1996) e Damelio (2011).

\subsection{SIPOC}

O SIPOC é uma ferramenta que busca identificar todos os elementos relevantes de um projeto antes do início do mesmo. Essa ferramenta exibe as inter-relações existentes no processo, evidenciando suas interfaces e o impacto destas interfaces na qualidade do output, contribuindo assim para o desenvolvimento de uma visão da organização voltada para processos.

O elemento fornecedor (S) do diagrama SIPOC, representa os indivíduos, departamentos ou organizações que provém materiais, informações ou recursos que serão trabalhados nos processos em análise. As entradas (I) representam as informações ou materiais fornecidos. O processo $(\mathrm{P})$ envolve os passos ou atividades que transformam as entradas em produto ou serviço final. As saídas (S) se referem aos serviços ou produtos finais que são resultantes do processo. Os clientes (C) são os indivíduos, departamentos ou organizações que recebem as saídas do processo.

A Matriz SIPOC é vista na Figura 1.

Figura 1 - Matriz SIPOC
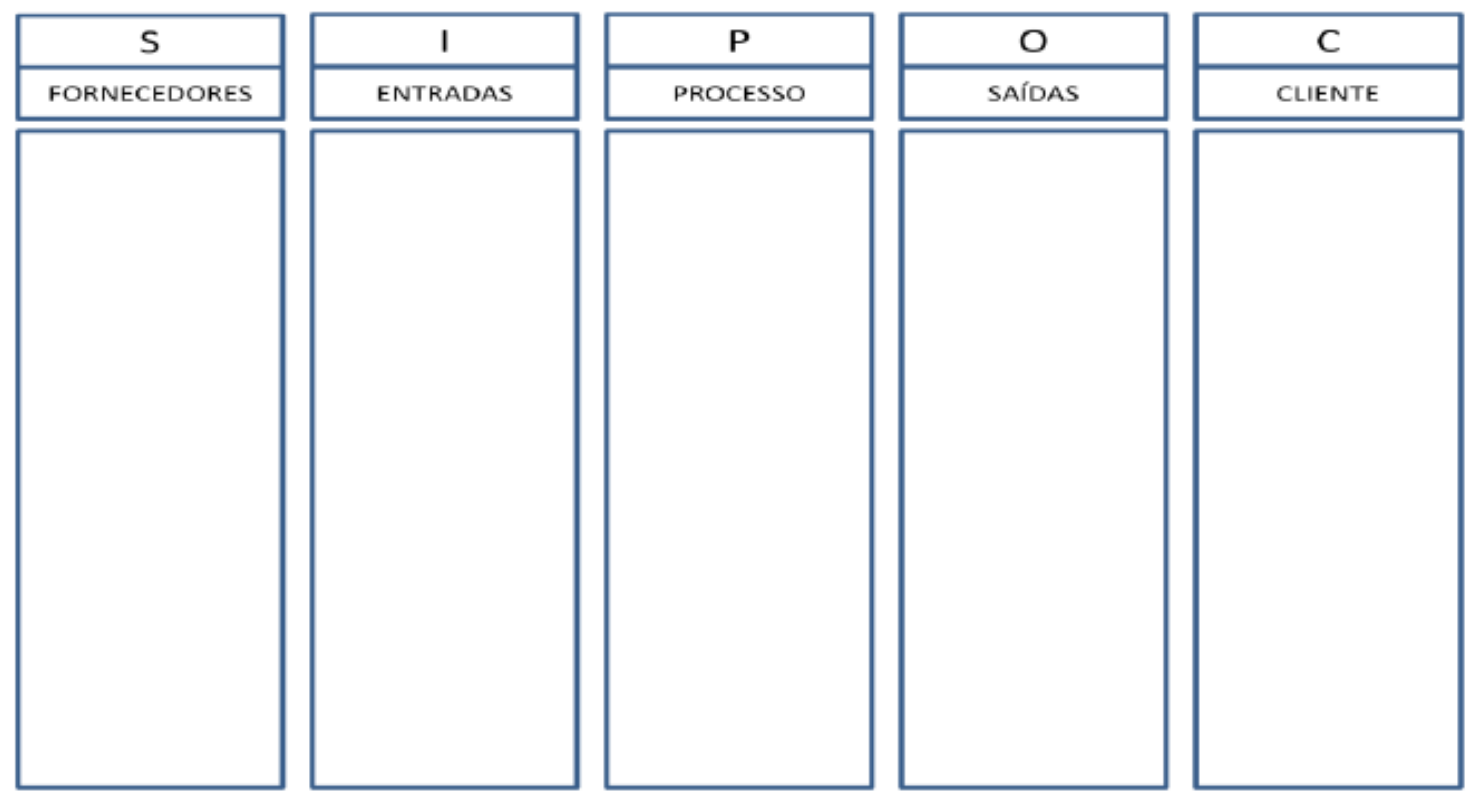

Fonte: http://www.docstoc.com

Para a elaboração da matriz SIPOC devem ser seguidos os seguintes passos: (i) Criar um nome para o processo; (ii) Criar uma área onde a equipe do projeto possa adicionar informações a Matriz SIPOC; (iii) Definir os subprocessos que fazem parte do processo mapeado; (iv) Identificar as Saídas; (v) Identificar os Clientes; (vi) Identificar as Entradas; (vii)Identificar os Fornecedores; (viii) Validar o processo com o responsável pelo projeto e outros stakeholders envolvidos.

\subsection{RELATIONSHIP MAP}

Um relationship map exibe as áreas (ou setores) de uma organização e a relação fornecedor-cliente existente entre elas. A leitura do mapa é realizada da esquerda para a direita, e este apresenta três componentes: Fornecedores, Organização e Consumidores. O relationship map exibe o trabalho ao nível da organização, porém não mostra explicitamente as atividades realizadas. As conexões entre os inputs (entradas) e 
outputs (saídas) que circulam entre os setores da empresa são também exibidos.

A utilização deste tipo de mapa deve-se fundamentalmente as seguintes razões: (i) Exibe os recursos recebidos pela empresa e o que ela produz; (ii) Ressalta o que cada parte da organização contribui para o output final; (iii) Torna visíveis algumas relações fornecedor- cliente; (iv) Ilustra os limites que o projeto deve ultrapassar para agregar valor; (v) Ajuda a orientar funcionários, fornecedores e outros stakeholders no entendimento em como o seu trabalho se encaixa no projeto da empresa; (vi) Mostra as partes e conexões que são afetadas por uma estratégia específica, iniciativa ou mudança de proposta.

Ao criar um relationship map é recomendável limitar o foco em um único serviço ou produto e iniciativa do cliente. Começar com a saída principal e com o principal cliente para essa saída auxilia na identificação do padrão geral a ser seguido para outros produtos ou serviços e clientes similares.

A metodologia de criação de um relationship map consiste em nove etapas, coforme apresentado a seguir: (i) Reservar uma grande área para a elaboração do mapa; (ii) Dividir essa área em dois espaços de trabalho; (iii) Criar um diagrama fornecedorconsumidor em um dos lados; (iv) Criar um relationship map básico no outro lado.

Estas quatro etapas estão exemplificadas na Figura 2, que mostra uma área de trabalho dividida em dois espaços de trabalho. Em um deles entrará o diagrama fornecedor-cliente que mostrará todas as relações e trocas comerciais realizadas entre os fornecedores e a organização, e no outro espaço ficará o relationship map.

Figura 2 - Área dividida em dois espaços de trabalho

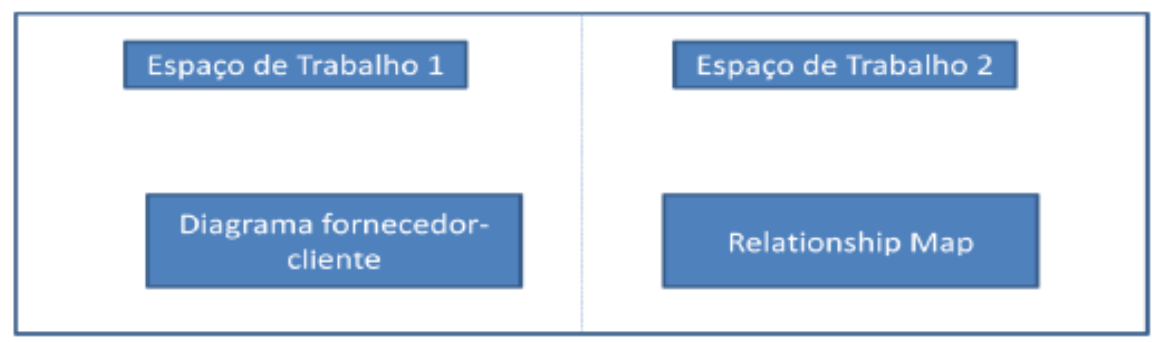

Fonte: Damelio (2011).

(v) Definir os clientes externos e as relações com estes; (vi) Definir os fornecedores externos e as relações com estes; (vii) Definir as relações entre fornecedores e clientes internos.

Estas etapas são realizadas para definir o diagrama fornecedor-consumidor ilustrado na Figura 3.

Figura 3 - Definição das relações fornecedores-consumidores

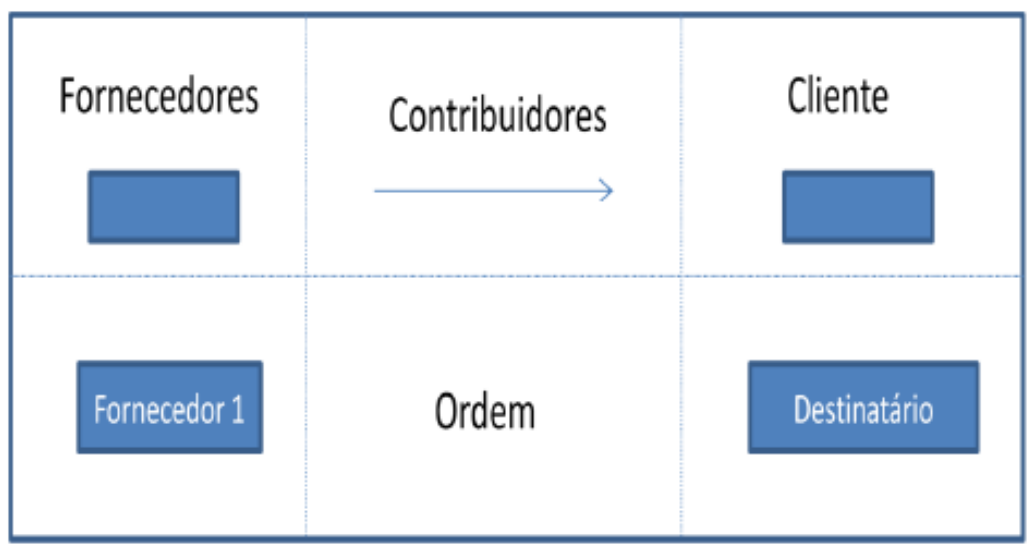

Fonte: Damelio (2011). 
(viii) Desenhar cada parte do diagrama fornecedor- cliente na coluna correspondente do relationship map; (ix) Identificar as entradas e saídas e fazer as conexões necessárias. Começar com as conexões entre organização e consumidor externo e depois identificar as conexões entre organização e fornecedor externo. Ao final realizar as conexões internas.

A execução destas últimas duas etapas pode ser visualizada na Figura 4 que mostra um exemplo de relationship map completo de uma oficina de automóveis. $\mathrm{Na}$ figura 4 também se observa todos os setores que tem participação no processo de negócio da oficina, desde o fornecedor até o cliente final, bem como todas as conexões, entradas e saídas existentes entre eles.

Figura 4 - Overview do procedimento de criação de um Relationship Map

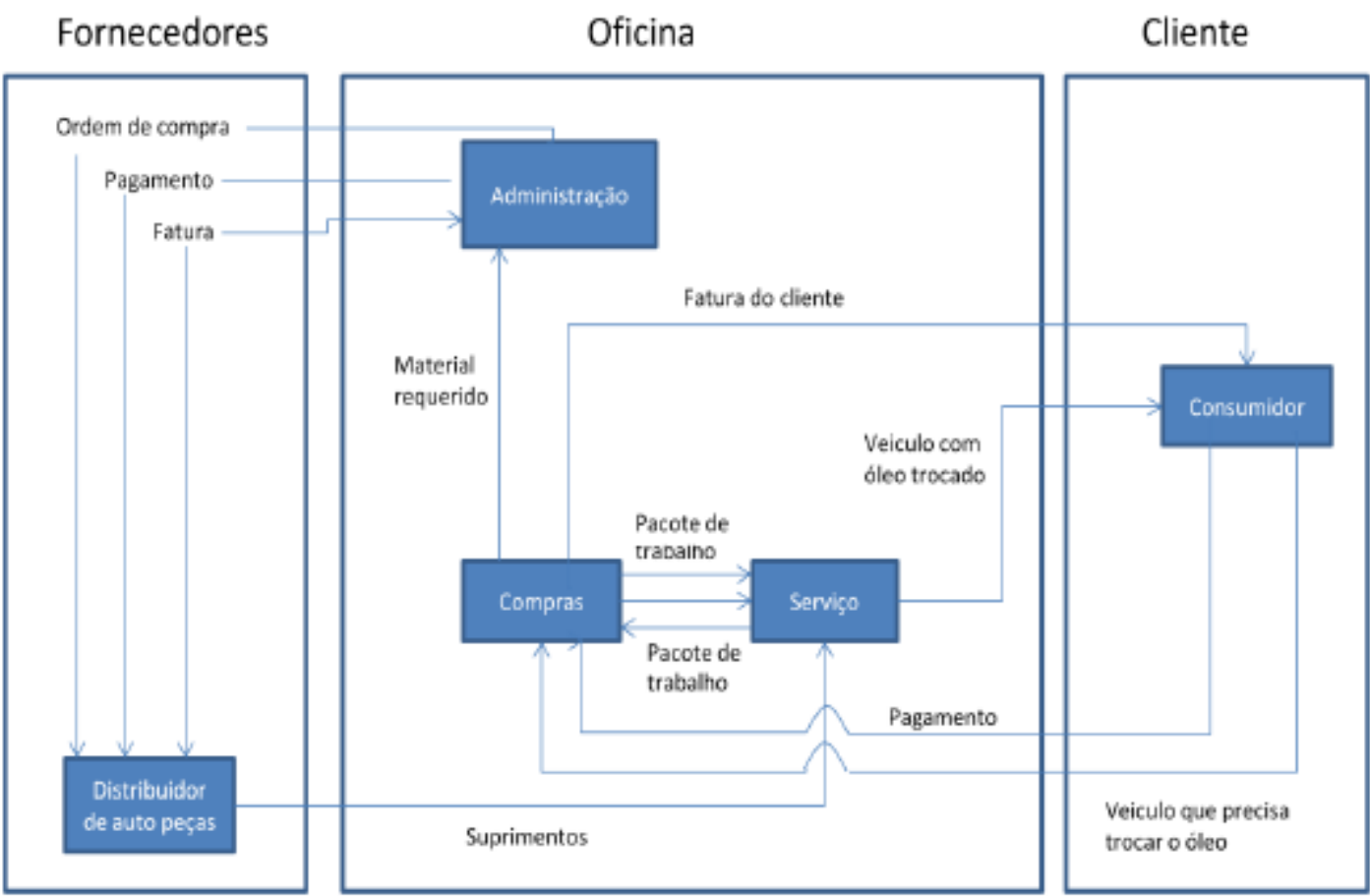

Fonte: Damelio (2011)

Um relationship map mostra como os setores de uma organização estão conectados. Toda função ou departamento é um fornecedor e um consumidor e, o mapa ajuda a identificar rapidamente qual papel o setor desempenha em determinado momento do processo. Assim, o relationship map responde a perguntas como: Quem são os consumidores da minha parte do negocio? Quais são os outputs que eu recebo? Quem são os fornecedores da minha parte do negócio? Quais os inputs que eu recebo? Como a minha parte do negocio se encaixa e se conecta com o resto da organização? Quais são as conexões críticas entre a minha área e o resto da organização?

Toda empresa tem algum tipo de fluxo de trabalho. Esse fluxo de trabalho começa e termina com um cliente externo e abrange várias partes do negócio. Depois de tornar visíveis as relações entre essas partes, as áreas da organização devem estar alinhadas para que operem integradamente na transformação de uma ordem de produção em um produto ou serviço. 


\subsection{Cross-Functional Process Map}

Um mapa cross-functional ilustra o fluxo de trabalho de uma organização. Um fluxo de trabalho consiste em um conjunto de atividades inter-relacionadas que seguem um determinado caminho enquanto transforma os inputs do projeto em outputs. $\mathrm{O}$ relationship map apresenta somente as partes da organização e suas conexões, o mapa cross-functional entra no detalhe do fluxo e mostra o trabalho que é realizado em cada parte.

A utilização deste tipo de mapa deve-se fundamentalmente aos seguintes motivos: (i) Mostra os limites (início e fim) e todo o fluxo de trabalho; (ii) Simultaneamente mostra a atividade e aonde essa atividade é realizada dentro da empresa; (iii) Torna visível a relação entre fornecedores e consumidores; (iv) Destaca os handoffs da empresa; (v) Identifica padrões no fluxo de trabalho; (vi) Ressalta os pontos de contato com os consumidores do fluxo.

Este tipo de mapa usa diversos símbolos, conforme pode ser visto na Figura 5. Caixas representam as atividades do fluxo de trabalho e caixas sombreadas quando se tem um fluxograma separado para a atividade. Usam-se setas para indicar as entradas e saídas de cada atividade. As setas devem passar por cima ou por baixo das outras e nunca se interceptar. Losangos indicam as decisões.

A metodologia de criação de um cross-functional map consiste em cinco etapas: (i) Reservar uma grande área para a elaboração do mapa; (ii) Desenhar uma faixa horizontal para cada entidade envolvida no processo; (iii) Posicionar as faixas, começando (de cima) pelo consumidor mais externo, seguido pelas entidades mais próximas do consumidor; (iv) Pedir a cada membro do grupo para escrever sua atividade na posição adequada.

Estas etapas estão ilustradas na Figura 5, que traz um exemplo da criação de um cross-functional map, mostrando as faixas horizontais para cada segmento da organização e as atividades que acontecem em cada um deles, permitindo assim a identificação das fronteiras do processo.

Figura 5 - Área de trabalho com as faixas desenhadas e atividade posicionadas adequadamente

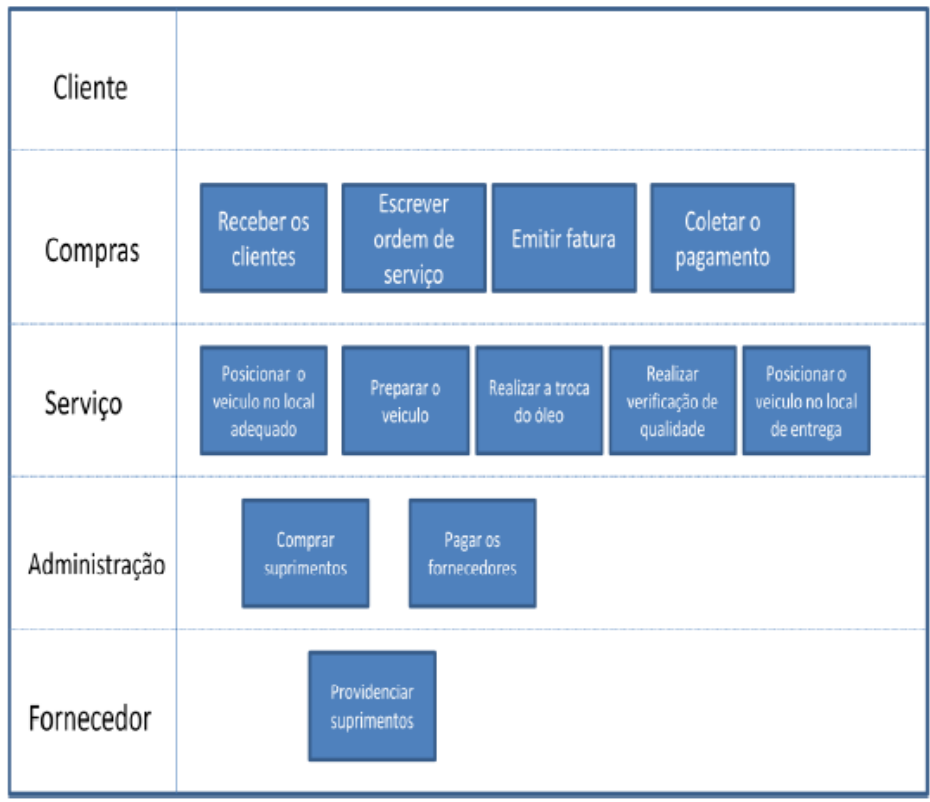

Fonte: Damelio (2011) 
(v) Colocar todos os inputs e outputs no fluxograma e fazer as conexões necessárias.

Esta última etapa pode ser visualizada nas figuras 6 e 7. Na Figura 6 pode-se observar um modelo de cross-functional map com todos os tipos de atividades e símbolos utilizados na elaboração deste tipo de mapa. A Figura 7 é o cross-functional map da oficina mecânica que estamos utilizando como exemplo, com as etapas do fluxo de trabalho posicionadas nas faixas correspondentes ao setor da organização em que elas ocorrem e todas as entradas e saídas envolvidas no processo.

Figura 6 - Cross-Functional Map

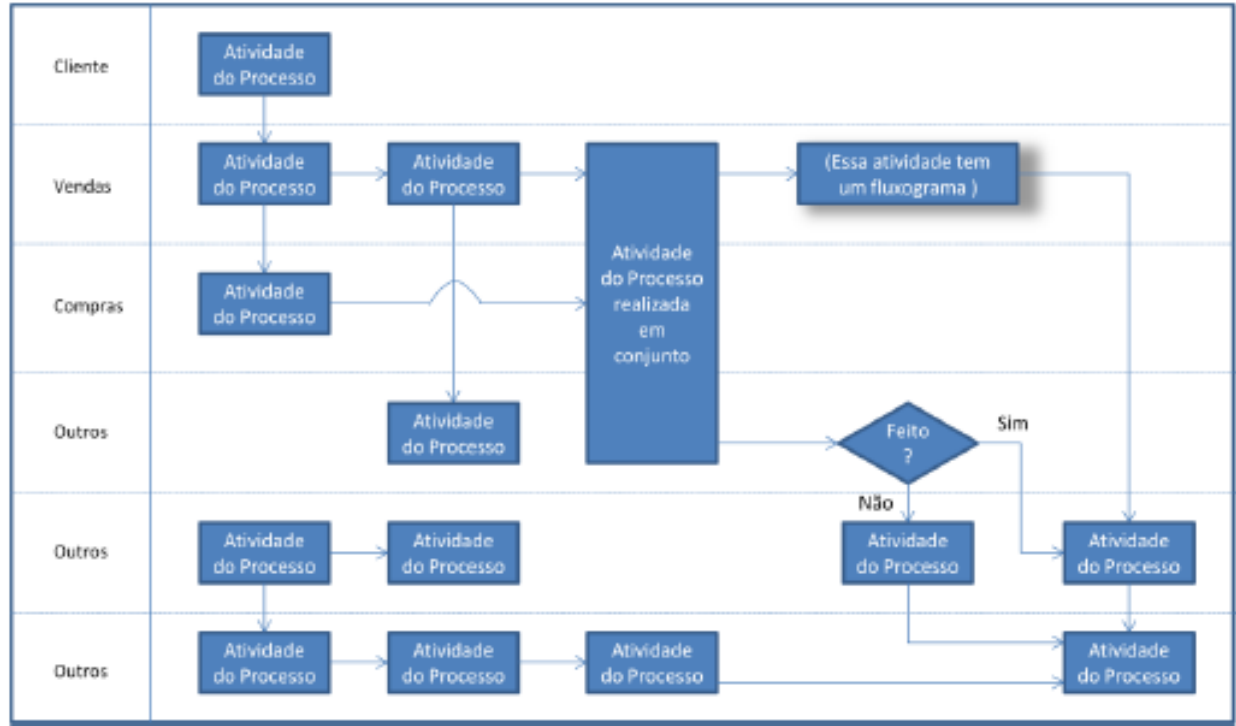

Fonte: http://www.rff.com

Figura 7 - Cross-Functional Map da oficina mecânica

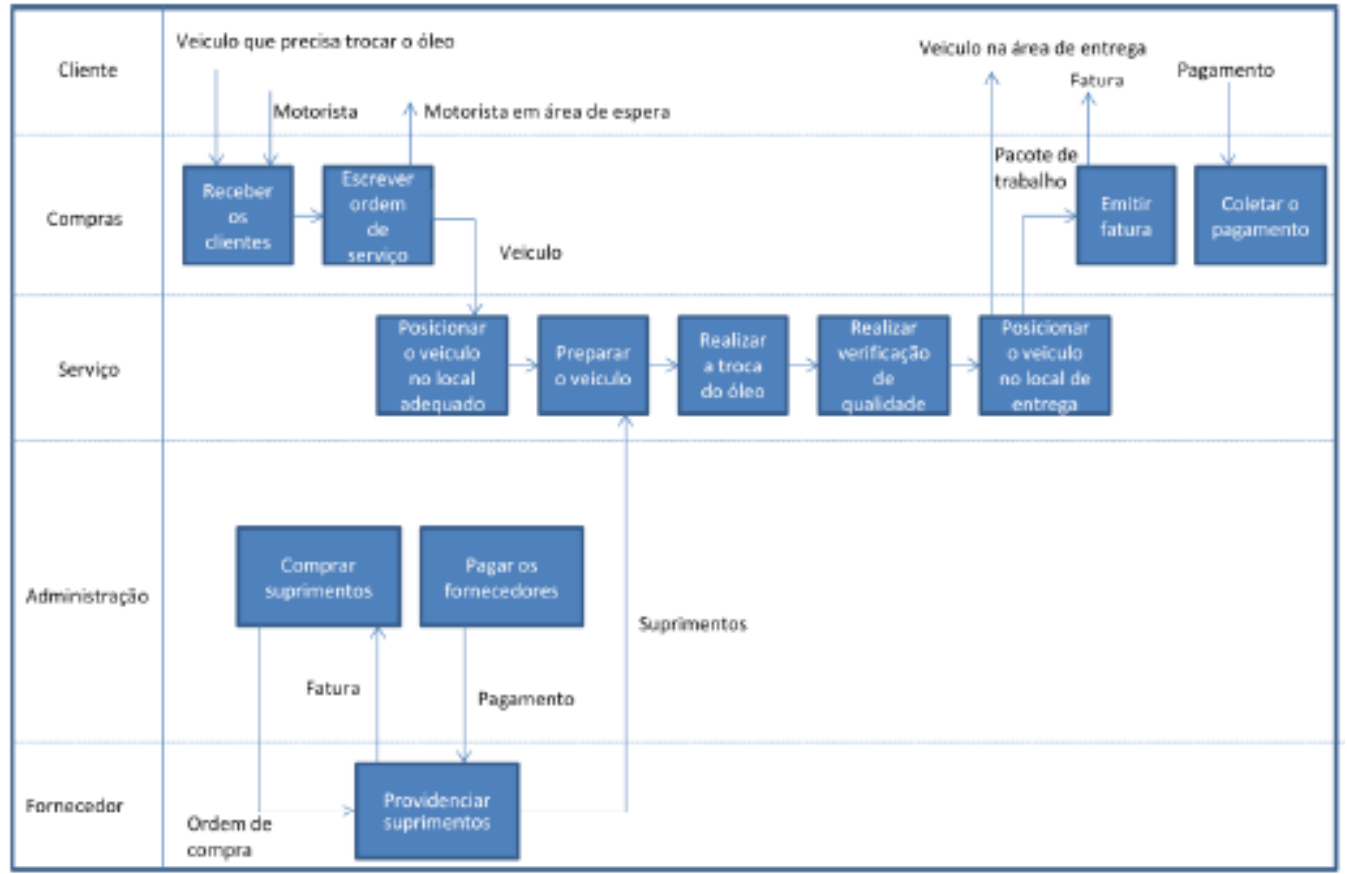

Fonte: Damelio (2011)

O cross-functional process map mostra os limites do fluxo de trabalho e destaca alguns componentes relevantes para esse fluxo. Enquanto o enfoque do 
relationship map está nas conexões entre as partes que compõe uma empresa, o crossfunctional process map mostra as atividades que ocorrem no interior dessas partes.

O cross-functional process map auxilia na resposta de perguntas como: Onde o fluxo de trabalho começa e termina? Quais atividades produzem o principal output desse fluxo? Qual a ordem em que as atividades ocorrem? Quais são os inputs necessários e outputs produzidos em cada atividade? O que desencadeia o fluxo de trabalho? Qual é o resultado contável do fluxo? Quais são as partes responsáveis por cada atividade? Quais são os handoffs e as interfaces entre as partes da organização e aonde elas ocorrem? Qual é o padrão do fluxo de trabalho?

\subsection{FLOWCHART}

Um flowchart (fluxograma) é uma representação gráfica de uma sequência de atividades usadas na produção de um output específico.

A utilização deste tipo de mapa deve-se fundamentalmente por conta dos seguintes motivos: (i) Mostra o que de fato ocorre nos subprocessos; (ii) Distingue atividades que agregam e não agregam valor; (iii) Torna visíveis as perdas geradas pelas atividades que não agregam valor como atrasos, movimentações, inspeções, retrabalhos, etc.

8.

Este tipo de mapa faz uso de diversos símbolos, conforme destacados na Figura

Figura 8 - Símbolos para Flowchart

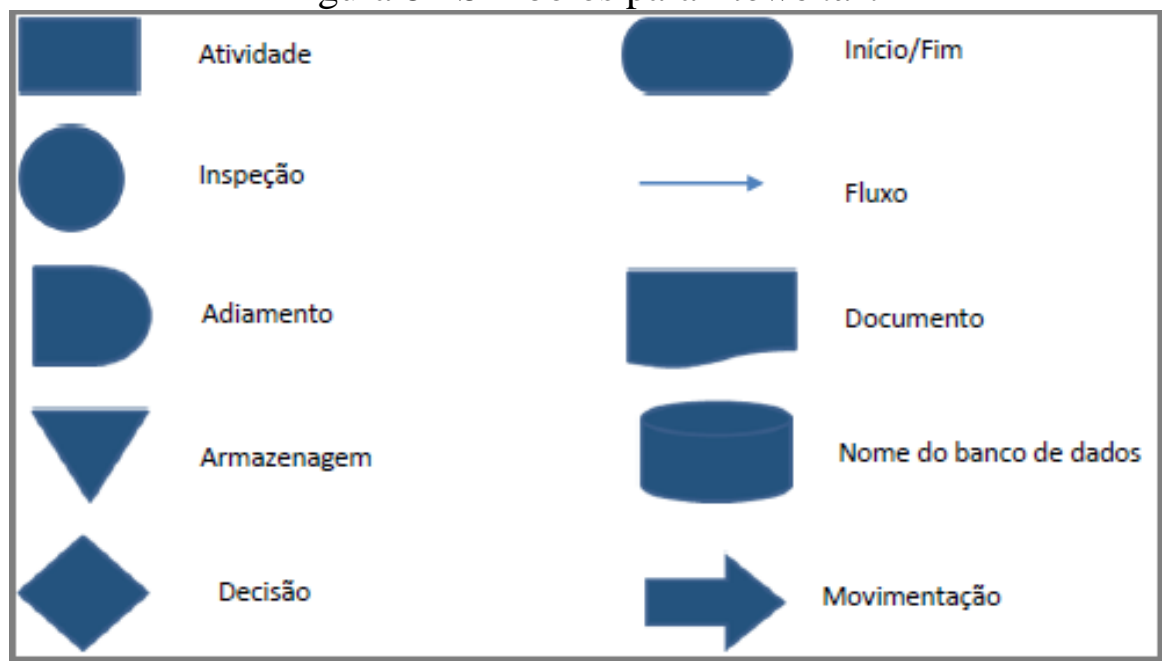

Fonte: Damelio (2011)

A metodologia de criação de um fluxograma consiste em seis etapas: (i) Definir o início e o fim do processo; (ii) Manter o fluxo da esquerda para a direita ou de cima para baixo; (iii) Utilizar os símbolos necessários; (iv) Manter os símbolos a uma mesma distância entre eles; (v) Evitar cruzamento de setas; (vi) Identificar os outputs do processo.

Estas etapas encontram-se ilustradas na Figura 9, que traz um exemplo de fluxograma de um dos processos da oficina mecânica com todas as atividades presentes e representadas com os símbolos adequados, e também seus outputs. 
Figura 9 - Flowchart de um processo da oficina mecânica

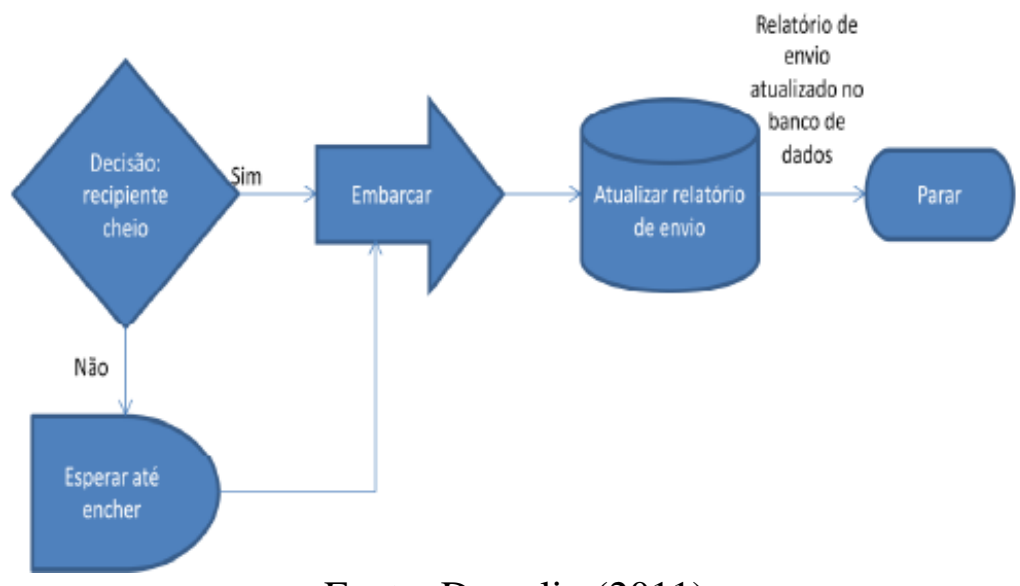

Fonte: Damelio (2011)

O fluxograma é uma representação gráfica de uma sequência de trabalho. Esse tipo de mapa normalmente é utilizado para compreender o trabalho no nível mais granular. Ele ajuda a identificar a complexidade das atividades, as perdas incorridas e as atividades que não agregam valor.

A maioria dos usuários dessa ferramenta tende a usar poucos símbolos e, assim, limitar a sua utilidade. Porém, o que torna o fluxograma útil é a utilização de símbolos suficientes que auxiliem no reconhecimento das perdas, atrasos e retrabalhos que ocorrem no processo.

\section{CASO NA ÁREA DE LOGÍSTICA DE UMA EMPRESA}

A empresa estudada está passando por uma reestruturação da sua área de logística com o objetivo de melhorar a eficiência e qualidade das atividades desse departamento. Uma das etapas dessa reestruturação é a instalação de um novo software para administrar e controlar os processos da área, como movimentação e aluguel de equipamentos, baixa de itens no sistema e devolução de equipamentos. Para que a instalação desse novo software fosse realizada, foi preciso mapear todos os processos da área de logística com a finalidade de buscar melhorias para esses processos e adaptá-los ao novo software.

Primeiramente, uma matriz SIPOC foi construída, onde foram definidos todos os fornecedores, entradas, subprocessos, saídas e clientes do macroprocesso em questão. A ferramenta mostrou-se de grande importância no auxílio à elaboração dos mapas dos processos da organização uma vez que proporciona um modo estruturado para a discussão do processo e gera um consenso entre os envolvidos antes que estes comecem a desenhar os diversos mapas dos processos. A Figura 10 exibe uma parte do SIPOC construído pela equipa da empresa. 
Figura 10 - SIPOC da empresa estudada

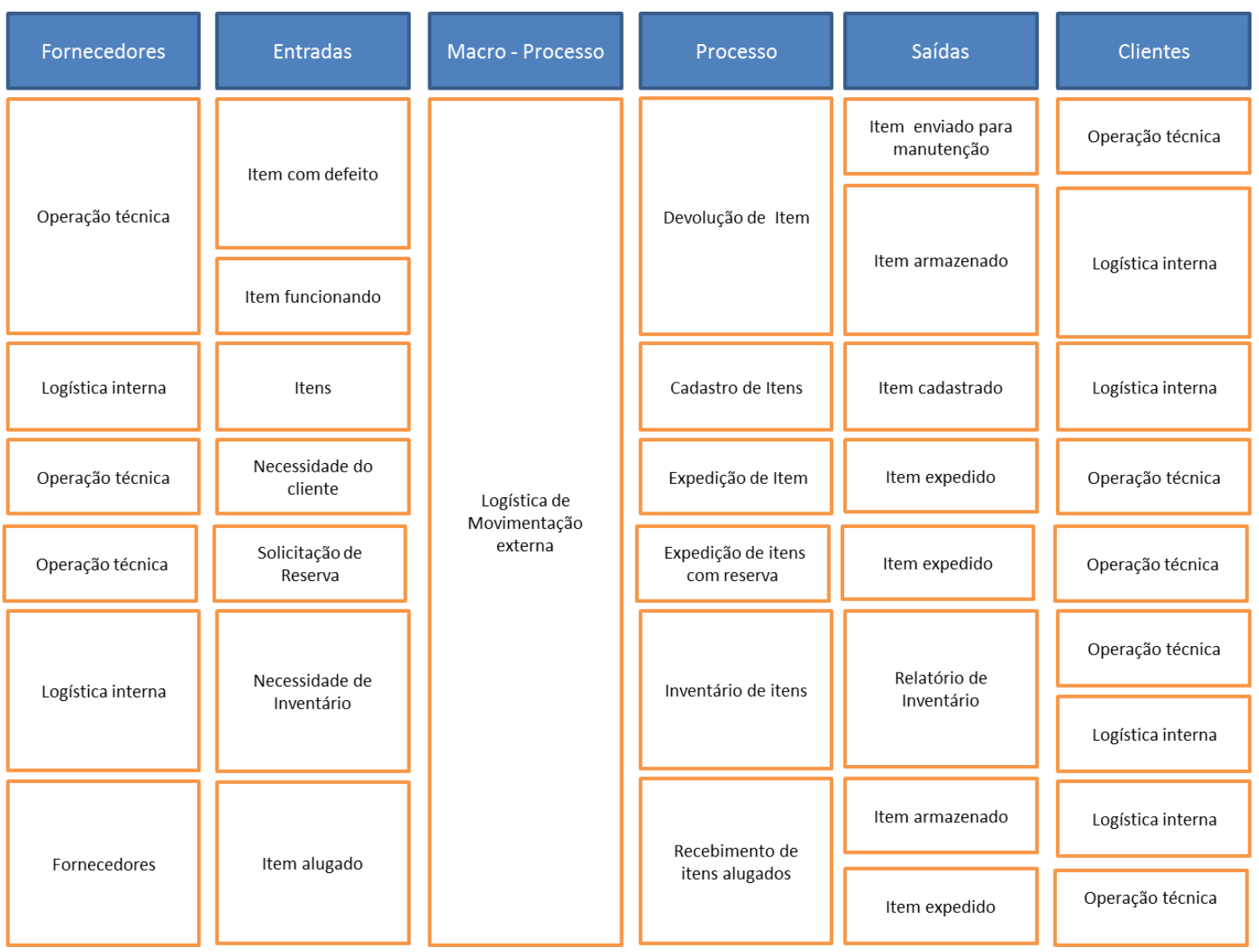

Após a elaboração do SIPOC, foram desenhados os mapas de cada subprocesso. A Figura 11 mostra um exemplo de um dos mapas que foram feitos pela equipe da empresa estudada. 
Figura 11 - Fluxograma da empresa estudada

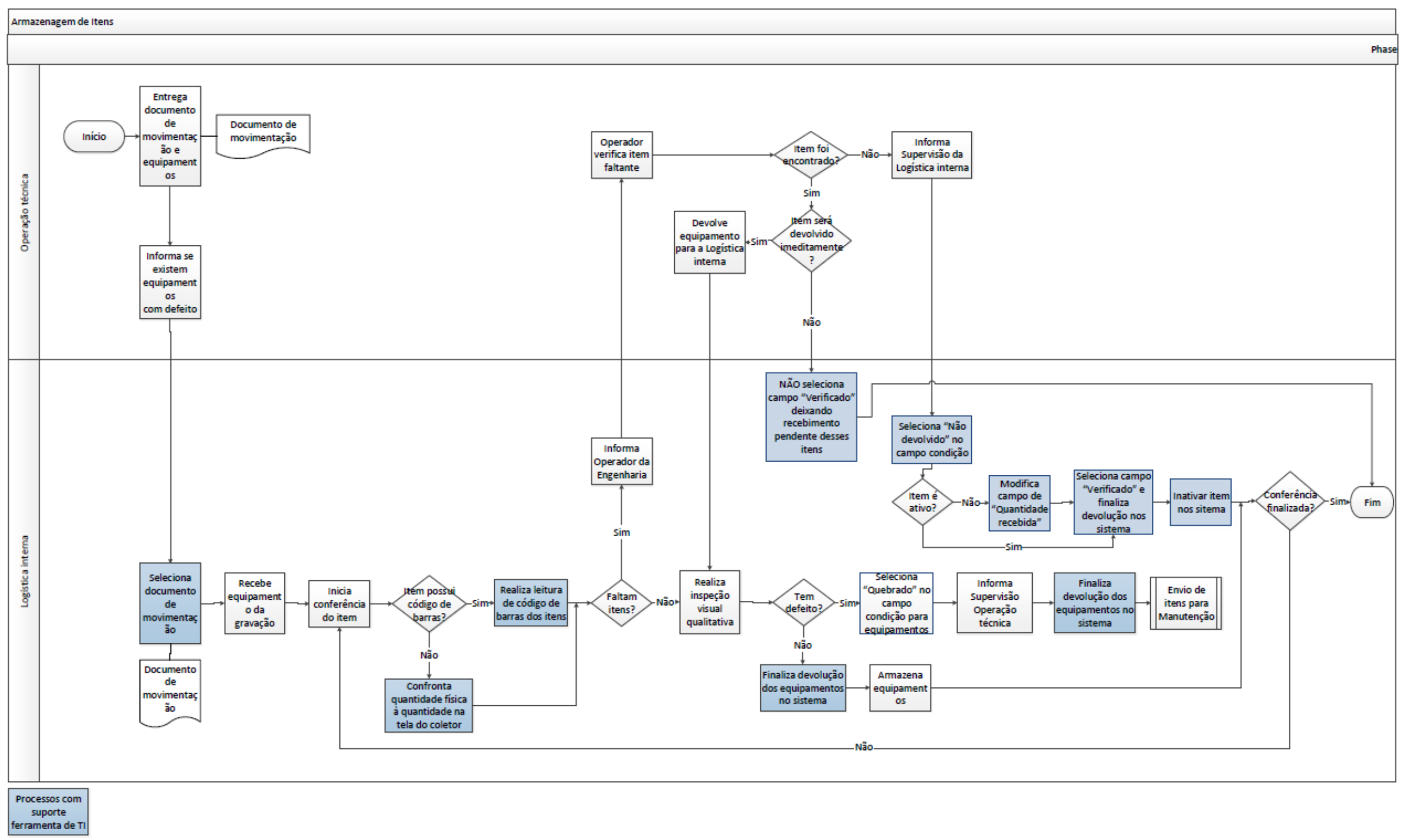


O mapa acima é de Recebimento e Armazenagem de Itens. Esse processo se passa em dois segmentos da empresa estudada: Operação técnica e Logística interna. Esse mapa mostra todas as atividades do processo em questão usando os símbolos adequados.

A empresa estudada usou em seu mapa uma mescla da metodologia do flowchart e do cross-functional map, pois detalha mais as atividades da área de logística, porém ainda aparecem no fluxograma tarefas realizadas por outras áreas.

A diferença entre o cross-functional map e o flowchart é que o primeiro mostra todo processo e todas as áreas envolvidas nele, já o flowchart é mais detalhado, descreve os subprocessos de uma área. Os dois tipos de mapa têm a sua importância: o crossfunctional para enxergar o processo como um todo e o flowchart para analisar todas as atividades de uma determinada área da organização e assim identificar onde ocorrem as perdas.

\section{CONCLUSÕES}

As crescentes mudanças no cenário de negócios geram a necessidade de procurar modelos de gestão que possam responder às novas condições de concorrência. Por meio dos estudos realizados pode-se perceber que o mapeamento de processos é uma ferramenta essencial para a melhoria e gestão de processos e projetos e tem grande importância para a Logística e a Engenharia de Produção. Com o mapeamento dos processos, é possível identificar os principais gargalos nos processos organizacionais, sejam estes de apoio ou de negócio, de forma a solucioná-los pelos meios mais seguros e eficientes, prezando sempre pela qualidade final do produto ou serviço prestado pela organização.

Existem diferentes tipos de mapas que podem ser utilizados de acordo com as finalidades e objetivos do projeto a ser realizado. $\mathrm{O}$ acompanhamento realizado junto à área de logística da empresa estudada apresentou benefícios como: documentação do conhecimento adquirido na vivência cotidiana pelos funcionários, formalização em linguagem comum das etapas que compõem os processos e identificação das interfaces críticas dos processos analisados. $\mathrm{O}$ envolvimento e comprometimento da equipe envolvida nas atividades também foi essencial para que os processos fossem observados, mapeados e analisados com a devida profundidade. Assim, melhorias foram identificadas e propostas pela própria equipe que realiza os processos. Desta forma, conclui-se que o mapeamento de processos, quando bem realizado, oferece inúmeros benefícios para as empresas e é um método que deve ser utilizado sempre que necessário.

\section{REFERÊNCIAS BIBLIOGRÁFICAS}

[1] ALOMAR, M., PASEK, Z.J. (2014). Linking supply chain strategy and processes to performance improvement, Procedia CIRP, v.17, p. $628-634$.

[2] ANJARD, R. (1998). Process mapping: a valuable tool for construction management and other professionals, ISSN 0263-2772.

[3] BIAZZO, S. (2002). Process mapping techniques and organizational analysis: lessons from sociotechnical system theory, Business Process Management Journal, v.8, n. 1, p. 42-52.

[4] DAMELIO, R. (2011). The Basics of Process Mapping, 2nd Edition, ISBN10:1363273764, CRC Press. 
[5] DAVENPORT, T.H. (1990). The New Industrial Engineering, Sloan Management Review, v. 31, n. 4, p. 11-27.

[6] GREASLEY, A. (2006). Using process mapping and business process simulation to support a process-based approach to change in a public sector organization, Technovation, v.26, p. 95-103

[7] HAMMER, M. (2002). Process Management and the future of Six Sigma, Sloan Management Review.

[8] HUNT, U.D. (1996). Process Mapping: How to Reengineer your Business Processes, ISBN-10:0471132810.

[9] KALMAN, H.K. (2002). Process Mapping: Tools, techniques \& critical success factors, Performance Improvement Quarterly, v. 15, n. 4, p. 57-73.

[10] KETTINGER, W.J., TENG, J.T.C., GUHA, S (1997). Business Process Change: A Study of Methodologies, Techniques, and Tools, MIS Quarterly, v. 21, n. 1, p. $55-80$

[11] MADISON, D. (2005). Process Mapping, Process Improvement and Process Management, ISBN-I3:9781932828047, Paton Press.

[12] OKRENT, M.D., VOKURKA, R.J. (2004). Process mapping in successful ERP implementations, Industrial Management \& Data Systems, v.104, n.8, p. 637 643.

[13] TRKMAN, P. (2010). The critical success factors of business process management, International Journal of Information Management, v.30, p. 125 134.

[14] ZEYNEP, T.S., OZALP, V., OZGEN, O. (2014). An outline of innovation management process: building a framework for managers to implement innovation, Procedia - Social and Behavioral Sciences, v.150, p. 690 - 699. 